\title{
The severity of non-planning impulsivity and cannabis use problems in distinguishing patients with synthetic cannabinoid use disorder and cannabis use disorder
}

\author{
Hanife Yilmaz Cengel ${ }^{1 \oplus}$, Muge Bozkurt ${ }^{2}{ }^{\oplus}$, Cuneyt Evren ${ }^{3 \oplus}$
}

${ }^{1}$ Istanbul Gelisim University, Faculty of Economics, Administrative and Social Sciences, Department of Psychology, Istanbul - Turkey ${ }^{2}$ Istanbul University, Faculty of Medicine, Department of Psychiatry, Istanbul - Turkey

${ }^{3}$ Bakirkoy Training and Research Hospital for Psychiatry Neurology and Neurosurgery, Research, Treatment and Training Center for Alcohol and Substance Dependence (AMATEM), Istanbul - Turkey

\begin{abstract}
Objective: The present study aims to identify whether there is a difference between the synthetic cannabinoid (SC) and cannabis use disorder groups in terms of impulsivity and problems arising from substance use, as well as to determine the variables that distinguish SC use disorder cases from cannabis use disorder.

Method: Fifty-two male patients with SC and 45 male patients with cannabis use disorder who were admitted to the AMATEM clinic of Bakirkoy Mental and Neurological Diseases Training and Research Hospital were included in the study. The participants were evaluated using the Barrat Impulsiveness Scale (BIS-11), Cannabis Use Problems Identification Test (CUPIT), Adult Cannabis Problems Questionnaire (CPQ), and Cannabis Withdrawal Scale (CWS).

Results: The SC use disorder group had higher rates of unemployment, suicide, and self-mutilation than the cannabis use disorder group. The SC use disorder group scored significantly higher than those with cannabis use disorder in BIS-11, CUPIT, CPQ total score and sub-scale scores, and CWS score.

Conclusion: It was shown in our study that cases with SC use disorder had a higher risk of impulsivity and problematic substance use than those with cannabis use disorder, and the CUPIT-B and non-planning impulsiveness sub-scales were also predictors of SC use disorder.
\end{abstract}

Keywords: Cannabis, impulsivity, problematic substance use, synthetic cannabinoid

\section{INTRODUCTION}

Cannabis is still the most common illicit psychoactive substance used worldwide. It usually started in early adolescence and is considered a gateway to other substances (1). Individuals who start using cannabis at an early age are more likely to later develop cannabis or other substance use disorders, deterioration in physical

How to cite this article: Yilmaz Cengel H, Bozkurt M, Evren C. The severity of non-planning impulsivity and cannabis use problems in distinguishing patients with synthetic cannabinoid use disorder and cannabis use disorder. Dusunen Adam The Journal of Psychiatry and Neurological Sciences 2021;34:41-49.

Correspondence: Hanife Yilmaz Cengel, Istanbul Gelisim University, Faculty of Economics, Administrative and Social Sciences, Department of Psychology - Turkey

E-mail: hanif_e@hotmail.com

Received: June 30, 2020; Revised: August 12, 2020; Accepted: January 07, 2021 
health, impairment in cognitive functions, lower academic and professional achievement, risky sexual behavior and a predisposition to crime (2). Although the rate of cannabis use has remained the same in recent years, the mental and physical problems associated with cannabis and the treatment admission rate are increasing in most of the countries due to the increase in tetrahydrocannabinol (THC) levels in cannabis and new consumption trends like dabbing (3).

Synthetic cannabinoids (SCs) are substances that were claimed to be natural products when they first appeared on the market and presented as an alternative to cannabis since they are more difficult to detect in urine (4). They were initially sold on the internet as a plant growth regulator or in drug stores claiming to be harmless, and rapidly became a significant problem with the increased use (5). According to European Monitoring Centre for Drugs and Drug Addiction data, SCs account for $45 \%$ of the newly reported substances so far, making them the largest new substance group (6). According to a study from Turkey, 1179 (98.3\%) out of 1200 herbal components delivered to the Institution of Forensic Medicine and Istanbul Narcotics Unit were found to contain SC (7). The type and amount of SC in mixtures are highly variable; sometimes a single product may contain multiple SCs that may interact with each other. This interaction leads to an even stronger effect and increased side effects (8). SCs affect the same endogenous cannabinoid receptors (CB1 and CB2) as cannabis. While $\triangle 9-T H C$, which is responsible for the psychoactive effects of cannabis, has a partial agonist effect on CB1 receptors, SCs show a full agonist effect (9). Moreover, SCs have a higher affinity for cannabinoid receptors and have a longer half-life than cannabis. Unlike cannabis, SC's metabolites have also high biological activity. The pharmacological effects of SCs have been shown to be 4 to 100 times greater than cannabis (10). Due to their lipophilic properties, SCs may also interact with non-cannabinoid receptors (4). Unlike cannabis, SCs do not contain cannabidiol and therefore do not show the antipsychotic and anxiolytic effects of cannabidiol (11). Although SCs would be expected to have similar effects to cannabis, it is clear that they have a much stronger effect, higher toxicity, more side effects, and more severe withdrawal symptoms compared to cannabis (12).

Impulsivity has been associated with substance use and related problems (13). Individuals with high levels of impulsivity have been shown to have higher rates of trying illicit substances, developing substance use disorders and relapse after treatment (14). It is also suggested that neurotoxicity-associated macrocellular structural defects in the brain or alterations in gene expression, synaptogenesis and neurogenesis together with substance use impair decision-making processes and inhibitory mechanisms, which in turn leads to impulsivity or increases existing impulsivity (15). In a study assessing the effects of SCs on cognitive functions in comparison with cannabis, researchers have reported significantly higher impulsivity -related executive dysfunction in SC users, such as rapid decision-making without considering negative outcomes, susceptibility to unplanned reactions, and inability to inhibit inappropriate stimuli (16).

Although there are numerous studies in the literature on clinical problems caused by SC use, particularly related to emergency unit admissions, issues such as interpersonal problems, risk of harm and addiction, psychological and motivational concerns, neglect of physical health, personal finances and social activities have been little investigated, and these have been little compared these with SC use disorder and cannabis use disorder in terms of impulsivity level and problems using various scales. The purpose of this study is to identify whether there is a difference between SC and cannabis use disorder groups in terms of impulsivity and problems arising from substance use, and variables that distinguish SC users from cannabis users.

\section{METHOD}

\section{Participants and Procedure}

The study was designed to include 52 male patients with SC use disorder admitted to the Alcohol and Substance Research, Treatment, and Education Center clinic of Bakirkoy Training and Research Hospital for Psychiatry, Neurology and Neurosurgery, and 45 male patients with cannabis use disorder applied to the Supervised Release Unit of the same hospital. The Ethics Committee approval was obtained before the study. The patients were informed and written informed consent was obtained. The Sociodemographic Data Form prepared by the researcher was used to collect information of the participants like age, gender, marital status, education level, employment status, criminal history, suicide and self-mutilation history, family history of psychiatric disorder and substance abuse, and disorders other than psychiatric disorders. The clinical psychiatric diagnosis was made through individual interviews according to DSM-5 criteria. Patients who had been using SC at least three days a week for at least 12 months and who did not consume 
other substances except tobacco during this period were recruited for SC use disorder group, and the patients among regular users who had been using cannabis at least three days a week for at least 12 months and who did not consume other substances except tobacco during this period were recruited for cannabis use disorder group. Urine toxicology tests were also performed in patient groups to confirm their substance use history and diagnosis. The participants then were administered the Barrat Impulsiveness Scale (BIS-11), Cannabis Use Problems Identification Test (CUPIT), Adult Cannabis Problems Questionnaire (CPQ), and Cannabis Withdrawal Scale (CWS). Illiterate and female patients were not included in the study. Patients with another substance use disorder or who found to be positive for a substance other than cannabis or SC in the drug metabolite screening test, who had mental retardation, history of head trauma, neurosurgery, epilepsy or any other major neurological disorder or another psychiatric disorder were excluded from the study. Two patients were excluded due to being illiterate, eleven patients were excluded due to being non-consent, five patients were excluded due to missing data and twelve patients were excluded due to having positive drug metabolite urine test for a substance other than SC or cannabis.

\section{Measures}

Sociodemographic Data Form: The sociodemographic and clinical characteristics of the study participants were assessed using the sociodemographic data form developed by the researchers. This form was used to gather information about age, gender, marital status, education level, employment status, criminal history, suicide and self-mutilation history, family history of psychiatric disorder and substance abuse, and disorders other than psychiatric disorders.

Barratt Impulsiveness Scale-11 (BIS-11): The BIS is an instrument filled by the patient and used to assess impulsiveness. It consists of thirty items and three factors: attention (attention deficit, cognitive instability), motor (motor impulsiveness, impatience), and non-planning (lack of self-control, intolerance to cognitive complexity) (15). The assessment of the BIS11 generates 4 different sub-scores: total score, nonplanning impulsiveness score, attentional impulsiveness score, and motor impulsiveness score. The higher the total BIS-11 score is, the higher the patient's impulsiveness level is. The Turkish validity and reliability study of BIS-11 was conducted by Gulec et al. (17).
Cannabis Use Problems Identification Test (CUPIT): The CUPIT is a short cannabis screening test consisting of 16 items. It can be used for various communities and all age groups of cannabis users, and is considered valid and reliable. It shows the cannabis use frequency and intensity in the last 12 months and the last 3 months, cannabis-induced problems, and the risk of harm and addiction. Three sub-groups are defined throughout the severity continuum: nonproblematic, risky, and problematic use (18). The scale has two factors: The CUPIT-A factor (items 1-9) and the CUPIT-B factor (items 10-16). Cronbach's alpha was 0.84 for CUPIT-A factor, 0.83 for CUPIT-B factor and 0.89 for CUPIT, when it was considered as a unidimensional scale. The Turkish version has been shown to effectively determine substance use problems in those with cannabis or SC use disorder (3).

Cannabis Problems Questionnaire (CPQ): The CPQ is a valid and reliable instrument used to identify problems associated with cannabis use, such as hazardous use, interpersonal problems, psychological and motivational concerns, and neglect of physical health, finances, and social activities (18). It was adapted from the alcohol problems questionnaire used to distinguish alcohol dependence and alcohol-related problems (19). The scale has a binary "Yes/No" format. The Turkish version of CPQ has been shown to effectively determine substance use problems in those with cannabis or SC use disorder (3).

Cannabis Withdrawal Scale (CWS): The CWS is a 19-items scale that can be used in clinical settings to measure cannabis withdrawal symptoms. The respondent self-assesses each symptom from 0 to 10 (from $0=$ none to $10=$ severe). The respondent also assesses the effect of each symptom on their everyday activities. Two scores can be derived from the scale: one for withdrawal intensity and one for the negative impact of withdrawal. Internal reliability (Cronbach's Alpha $=0.91$ ), test-retest stability (average intra-class correlation $=0.95$ ) and content validity analysis show that the CWS has excellent psychometric properties (20). The scale was translated by the authors of the present study and used in previous studies (3).

\section{Statistical Analysis}

SPSS Version 20.0 for Mac OSX was used for statistical analysis. In statistical evaluations, descriptive statistics (frequency and ratio) were used for socio-demographic variables. The Chi-square test was used to investigate the relationship between categorical variables and the Mann-Whitney U test was used for continuous variables 
of independent groups with non-normal distribution. Forward logistic regression analysis was conducted to evaluate the variables that distinguish the group with SC use disorder from the group with cannabis use disorder. BIS-11, CUBIT, CPQ and CWS were taken as independent variables. This model revealed that the variables that distinguish between those with SC use disorder and those with cannabis use disorder were CUPIT and BIS-11 scores. In the second Model, where CUBIT and BIS- 11 subscales were taken as independent variables instead of total scores, the severity of CUBIT-B and non-planning impulsiveness distinguish patients with SC use disorder from patients with cannabis use disorder. The results were evaluated at 0.05 level of significance.

\section{RESULTS}

The study sample included 45 patients between the ages of 18-65 diagnosed with cannabis use disorder and 52 patients between the ages of 18-65 diagnosed with SC use disorder according to DSM- 5 criteria. The mean age of the study participants was $27.58 \pm 6.22$. No statistically significant difference was found between the groups in terms of mean age, educational level, and marital status ( $>0.05)$ (Table 1). In terms of employment, the rate of unemployed among the SC group (51.9\%) was higher than that of cannabis group (8.9\%). The regular employment rate was lower among the SC group $(38.5 \%)$ compared to the cannabis group $(88.9 \%)$ $(\mathrm{p}<0.01)$ (Table 1). No significant difference was found between the groups in terms of admission to psychiatry clinic, alcohol and substance use history and history of psychiatric disorders among close relatives (Table 1). The rate of those who had attempted suicide was 19.2\% $(n=10)$ in the SC group and $4.4 \%(n=2)$ in the cannabis group; while the rate of self-mutilation was $51.9 \%$ $(n=27)$ in the SC group and $31.1 \%(n=14)$ in the cannabis group. Both values were significantly higher in the SC group than the cannabis group $(\mathrm{p}<0.05)$. In terms of the BIS-11 scores of the study sample, the SC group scored $17.87 \pm 4.19$ and the cannabis group scored $14.18 \pm 2.96$ in the attentional impulsiveness sub-scale; the SC group scored $24.58 \pm 6.19$ and the cannabis group scored $19.11 \pm 3.45$ in the motor impulsiveness sub-scale; the SC group scored $28.25 \pm 5.19$ and the cannabis group scored $22.49 \pm 4.21$ in the non-planning impulsiveness sub-scale; and the SC group scored $70.69 \pm 13.16$ and the cannabis group scored $55.78 \pm 8.23$ in total BIS-11. The SC group scored significantly higher in all sub-scales as well as total BIS-11 ( $<<0.001)$ (Table 2). In terms of the CUPIT scores of the study sample, the SC group scored $35.06 \pm 9.57$ and the cannabis group scored $25.76 \pm 9.30$ in the CUPIT-A sub-scale; the SC group scored $11.94 \pm 4.95$ and the cannabis group scored $6.31 \pm 3.91$ in the CUPIT-B sub-scale; and the SC group scored $47.00 \pm 13.04$ and the cannabis group scored $32.07 \pm 11.70$ in total CUPIT. The SC group scored significantly higher in all sub-scales as well as total CUPIT $(\mathrm{p}<0.001)$ (Table 2). In terms of the CPQ scores of the study

Table 1: Comparison of groups by sociodemographic variables

\begin{tabular}{|c|c|c|c|c|c|c|}
\hline & & $\begin{array}{c}\text { Synthetic } \\
\text { cannabinoid } \\
(n=52)\end{array}$ & & $\begin{array}{c}\text { Cannabis } \\
(n=45)\end{array}$ & & \\
\hline & Mean & SD & Mean & SD & $\mathbf{z}$ & $\mathbf{p}$ \\
\hline Age (years) & 27.03 & 6.08 & 28.84 & 6.37 & 3.286 & 3.286 \\
\hline \multirow[t]{2}{*}{ Duration of education (years) } & 9.00 & 2.63 & 8.97 & 3.25 & 5.717 & 0.057 \\
\hline & $\mathbf{n}$ & $\%$ & $\mathbf{n}$ & $\%$ & $\chi^{2}$ & $\mathbf{p}$ \\
\hline Marital status $^{+}$ & & & & & 7.445 & 0.230 \\
\hline Single & 31 & 59.6 & 24 & 53.3 & & \\
\hline Married & 17 & 32.7 & 15 & 33.3 & & \\
\hline Divorced, widow, separate & 4 & 7.7 & 6 & 13.3 & & \\
\hline Employment status $^{+}$ & & & & & 53.20 & 0.001 \\
\hline Unemployed & 27 & 51.9 & 4 & 8.9 & & \\
\hline Regular employed & 20 & 38.5 & 40 & 88.9 & & \\
\hline Irregular employed & 5 & 9.6 & 1 & 2.2 & & \\
\hline Suicide attempt & 10 & 19.2 & 2 & 4.4 & 4.865 & 0.027 \\
\hline Self-mutilation & 27 & 51.9 & 14 & 31.1 & 4.282 & 0.039 \\
\hline
\end{tabular}

\footnotetext{
z: Mann-Whitney U Test
} 
sample, the SC group scored $5.90 \pm 2.32$ and the cannabis group scored $3.33 \pm 2.76$ in the CPQ-A sub-scale; the SC group scored $3.69 \pm 1.55$ and the cannabis group scored $1.78 \pm 1.46$ in the CPQ-B sub-scale; the SC group scored $2.15 \pm 0.87$ and the cannabis group scored $1.78 \pm 1.20$ in the CPQ-C sub-scale; and the SC group scored $11.75 \pm 3.77$ and the cannabis group scored $6.89 \pm 4.42$ in total CPQ. The SC group scored significantly higher in all sub-scales as well as total CPQ $(\mathrm{p}<0.001)$ (Table 2). In terms of the CWS scores of the study sample, the SC group scored $69.58 \pm 25.63$ and the cannabis group scored $32.36 \pm 26.91$ in CWS. The SC group scored significantly higher compared to the cannabis group in CWS $(p<0.001)$ (Table 2$)$. The logistic regression analysis revealed that the variables discriminating those with SC use disorder from those with cannabis use disorder were CUPIT and BIS-11 scores. In the second logistic regression analysis, where subscales instead of total scores of the scales were taken as independent variables, the CUPIT-B and non-planning impulsiveness sub-scales were found to be predictors (Table 3 ).

\section{DISCUSSION}

A comparison between the groups in terms of employment showed that the unemployment rate among the SC group (51.9\%) was higher than that of cannabis group (8.9\%), while among the SC group the regular employment rate was lower $(38.5 \%)$ compared to the cannabis group (88.9\%). In one study from Turkey investigating clinical characteristics and laboratory results of patients admitted to a outpatient clinic due to SC use, the researchers found $42.4 \%(n=67)$ of the patients were employed regularly, $51.9 \%(n=82)$ were employed irregularly, and $5.7 \%(\mathrm{n}=9)$ were unemployed (21). Moreover, we found the unemployment rate to be

Table 2: Comparison of groups by scale scores

\begin{tabular}{|c|c|c|c|c|c|c|}
\hline & & $\begin{array}{c}\text { Synthetic } \\
\text { Cannabinoid } \\
(n=52)\end{array}$ & & $\begin{array}{c}\text { Cannabis } \\
(n=45)\end{array}$ & & \\
\hline & Mean & SD & Mean & SD & $\mathbf{z}$ & $\mathbf{p}$ \\
\hline BIS-11 & 70.69 & 13.16 & 55.78 & 8.23 & 6.78 & $<0.001$ \\
\hline Attentional impulsiveness & 17.87 & 4.19 & 14.18 & 2.96 & 5.05 & $<0.001$ \\
\hline Motor impulsiveness & 24.58 & 6.19 & 19.11 & 3.45 & 5.46 & $<0.001$ \\
\hline Non-planning impulsiveness & 28.25 & 5.19 & 22.49 & 4.21 & 5.94 & $<0.001$ \\
\hline CUPIT & 47.00 & 13.04 & 32.07 & 11.70 & 5.90 & $<0.001$ \\
\hline CUPIT-A & 35.06 & 9.57 & 25.76 & 9.30 & 4.84 & $<0.001$ \\
\hline CUPIT-B & 11.94 & 4.95 & 6.31 & 3.91 & 6.25 & $<0.001$ \\
\hline CPQ & 11.75 & 3.77 & 6.89 & 4.42 & 5.85 & $<0.001$ \\
\hline CPQ-A & 5.90 & 2.32 & 3.33 & 2.76 & 4.99 & $<0.001$ \\
\hline CPQ-B & 3.69 & 1.55 & 1.78 & 1.46 & 6.22 & $<0.001$ \\
\hline CPQ-C & 2.15 & 0.87 & 1.78 & 1.20 & 1.74 & 0.086 \\
\hline CWS & 69.58 & 25.63 & 32.36 & 26.91 & 6.97 & $<0.001$ \\
\hline
\end{tabular}

Mann-Whitney U Test, CUPIT: Cannabis Use Problems Identification Test, CPQ: Cannabis Problems Questionnaire, CWS: Cannabis Withdrawal Scale

Table 3: Variables identifying synthetic cannabinoid users in forward logistic regression model

\begin{tabular}{|c|c|c|c|c|c|c|c|c|}
\hline & \multirow[t]{2}{*}{ B } & \multirow[t]{2}{*}{ S.E. } & \multirow[t]{2}{*}{ Wald } & \multirow[t]{2}{*}{ df } & \multirow[t]{2}{*}{$\mathbf{p}$} & \multirow[t]{2}{*}{ Odds ratio } & \multicolumn{2}{|c|}{ 95\% C.I. } \\
\hline & & & & & & & Lower & Upper \\
\hline \multicolumn{9}{|l|}{ Step 1} \\
\hline CUPIT & 0.058 & 0.022 & 6.660 & 1 & 0.010 & 1.059 & 1.014 & 1.107 \\
\hline BIS-11 & 0.091 & 0.027 & 11.428 & 1 & 0.001 & 1.095 & 1.039 & 1.155 \\
\hline \multicolumn{9}{|l|}{ Step 2} \\
\hline CUPIT-B & 0.222 & 0.063 & 12.443 & 1 & $<0.001$ & 1.248 & 1.104 & 1.412 \\
\hline NP Imp. & 0.201 & 0.060 & 11.424 & 1 & 0.001 & 1.223 & 1.088 & 1.375 \\
\hline
\end{tabular}

Nagelkerke $\mathrm{R}^{2}$ : Step 1=0.482, Step 2=0.512. CUPIT: Cannabis Use Problems Identification Test, CPQ: Cannabis Problems Questionnaire, CWS: Cannabis Withdrawal Scale, C.I.: Confidence interval, S.E.: Standard error 
considerably high among those with SC use disorder. Consistent with the results obtained from CUPIT and $\mathrm{CPQ}$ in our study, these findings suggest that individuals in the SC use disorder group experience more problems in professional life than the cannabis use disorder group. These findings may be explained with more pronounced negative clinical effects and withdrawal symptoms seen in SCs compared to cannabis. Also, the significantly higher BIS-11 scores of the SC group may explain the problems in professional life due to the more impulsive trait of this group. Unemployment is an important dimension of social exclusion (22) and because the other dimensions of the social exclusion are not included, unemployment bears the burden of social exclusion in our study.

The rate of those who had attempted suicide was $19.2 \%(n=10)$ in the SC group and $4.4 \%(n=2)$ in the cannabis group; while the self-mutilation rate was $51.9 \%$ $(n=27)$ in the SC group and $31.1 \%(n=14)$ in the cannabis group. Both values were significantly higher in the SC group than in the cannabis group. Consistent with our findings, an increase in suicides was reported following SC use (23). A 23-year-old man with no psychiatric disorder history was reported to have committed suicide by stabbing himself after using a high concentration of AM-2201(1-[5-fluoropentyl]-3[1-naphthyl]indole) (24) and another case of suicide by jumping from height after using an unknown SC derivative (25). A person with a previous history of paranoid schizophrenia was reported to have engaged in self-mutilation characterized by right jugular vein cut associated with intense agitation and anxiety after SC use (26). Psychotic symptoms accompanied by agitation and aggression are more common among SC users. In a study investigating aggressive behavior in a psychiatry clinic, participants were grouped into SC-only users, cannabis-only users, both SC and cannabis users, and those who did not use either. The group with the most prevalent psychotic symptoms was found to be those using SC only, followed by those using both SC and cannabis. Similarly, the rate of displaying agitation was significantly higher in SC-only users, while aggression was the most common among both SC and cannabis users (27). The researchers investigated the risk-taking and violent behavior in 9th-12th grade students with SC and cannabis use, and those experimented with SC were found to display more physical and sexual violence during dating, to engage in fights, injure others, and to have a higher rate of carrying a weapon (28). The rate of suicide or attempted self-mutilation was found to be associated with SC use in some studies $(29,30)$. The sensitivity of CB1 receptors in the prefrontal cortex is believed to be effective in the pathophysiology of suicidal behavior (31). Considering that SCs are 4 to 100 times stronger than cannabis, the higher rate of suicide and self-mutilative behavior among SC users seems to be consistent with the literature. Higher impulsivity is related to suicidal and self-mutilative behavior (32). It was observed in our study that SC users had higher impulsivity. It is not known whether those with higher impulsivity have higher SC use or whether impulsivity is increased due to the effects of SCs on the prefrontal and orbitofrontal cortex; but higher impulsivity in these individuals may be associated with higher suicide rate and self-mutilative behavior.

In the present study, the impulsivity levels of the groups were measured using BIS-11, and it was found that the SC group had significantly higher scores in all sub-scales and total BIS-11 than the cannabis group. In a study comparing alcohol, opioid, and SC use disorders in terms of violent behavior and childhood trauma, researchers used BIS-11 and found no difference between the groups with regard to impulsivity; however, verbal and physical violence was more common among those with SC use disorder than other groups and commitment to crime was also higher for SC users (33). It is suggested that substances lead to impulsivity or increase existing impulsivity due to their acute and chronic effects (34). Substance use itself may increase maladaptive behaviors, either due to their direct, acute effects or the long-term sequelae of substance use (35). Also, the effect of substance use on decision-making may lead to a continuous and unplanned increase in substance use. Impairments in inhibitory mechanisms lead to disinhibition according to substance use, i.e. impulsive behaviors (36). In a study assessing the effects of SCs on cognitive functions comparatively with cannabis, researchers have reported significantly higher impulsivity-related executive function impairment in SC users, such as rapid decision-making without considering its negative outcomes, susceptibility to unplanned reactions, and inability to inhibit inappropriate stimuli (16). In another study, impairments in inhibitory control mechanisms were shown to be more prevalent in SC users compared to cannabis users (37). The higher level of impulsivity among SC users in our study may be due to the high neurotoxic effects of the substances used. Sometimes the synergistic effect of multiple SC types in a single product may cause increased neurotoxicity, and additives other than SC may aggravate this effect. The NPI dimension of impulsivity, which can be defined as 
the tendency to choose a smaller, more immediate reward rather than a larger, more delayed reward and focused on the "present orientation" with a "lack of planning for the future and foresight" (38), distinguished patients using SC from cannabis users in the present study. In the previous studies with the substance use population, the NPI predicted also the cannabis use among heavy drinkers, or problem drinking among alcohol users (39) and distinguished opioid addicts from healthy controls (33). Taken together with the results of other studies, the fact that SC use disorder, which is associated with more problems than cannabis use, was predicted by NPI leads to the idea that NPI is associated with more problematic substance use.

In our study, patients with SC use disorder obtained significantly higher scores in CUPIT total score, CUPIT-A, and CUPIT-B sub-scales; CPQ total score, CPQ-A, and CPQ-B sub-scales; and CWS $(\mathrm{p}<0.001)$ compared to those with cannabis use disorder. In comparison to those with cannabis use disorder, people with SC use disorder have significantly more problems associated with the SC use such as higher substance use frequency and intensity within the last 12 and within the last 3 months, increased risk of harm and addiction, interpersonal problems, psychological and motivational concerns, and neglect of physical health, finances, and social activities. Also, SC users report significantly higher symptoms and effects of withdrawal on daily life compared to cannabis users. To our knowledge, there is no study in the literature comparing SC users and cannabis users by means of CUPIT or CPQ. In a previous study investigating risky behaviors in SC and cannabis users in four fields (substance use, injury/violence, mental health, and sexual health), researchers found that SC users were significantly more likely to engage in risky behaviors in the substance use and sexual risk fields compared to cannabis users (33). The logistic regression analysis revealed that one of the variables that distinguish the SC group and the cannabis group was CUPIT-B. This sub-scale measures the problems associated with substance use, and this supports the findings of our study indicating more serious problems (unemployment, suicide, self-mutilation) caused by SC use. SC users are observed to experience more severe withdrawal symptoms and have more difficulty in quitting substance use in clinical practice than cannabis users, which is consistent with the results of the scales used in our study. The sociodemographic data obtained in our study showed that SC users had a higher unemployment rate, self-mutilative behavior, suicide attempt and impulsivity, which is consistent with the data based on CUPIT and CPQ scores. Present study has several limitations. First of all, the fact that all participants of the study were male prevents us from commenting on female SC use disorder cases. Also, we could not explore impulsivity and problematic substance use in adolescent patients, since only those aged between 18-65 years were included in the study. Another limitation of our study is that SCs have wide variety and there was a lack of data regarding different effects of these varieties and which varieties were used by the patients. One of the most significant limitations of our study is that it included patients who were self-hospitalized in the SC use disorder group and patients who were under probation in the cannabis use disorder group, a fact which may have caused different substance use characteristics between these two groups.

To conclude, in the present study, unemployment, suicide and self-mutilation rates were higher in the SC use disorder group compared to the cannabis use disorder group; the SC use disorder group scored significantly higher on the BIS-11, CUPIT, CPQ total score and sub-scale scores and CWS score than those with cannabis use disorder, resulting in that SC use disorder causes a higher risk of impulsivity and problematic substance use compared to cannabis use disorder. It was also shown in our study that the CUPIT-B and non-planning impulsiveness sub-scales in particular were predictors of SC use disorder. The higher level of impulsivity and more problems associated with substance use among SC users may have a number of possible causes. SCs affect several receptors that cannabis does not, which may explain why SC users experience more severe neurotoxic effects, leading to a greater number of withdrawal symptoms, and causing more problems compared to cannabis. The neuroprotective effects of cannabidiol found in cannabis have been demonstrated (11). Therefore, it may be suggested that SC users, who do not experience cannabidiol's neuroprotective effects, may have more severe impairments in inhibitory processes, higher impulsivity, and a higher rate of self-mutilation and suicide attempts. Additionally, the heterogeneous nature of SCs products may impair inhibitory functions. The higher frequency and amount of substance use may cause more adverse effects on cognitive functions and more problems associated with substance use. Determining substance-related problems caused by SC use disorder might help us understand physical, functional, communicational, and social issues of users, 
identify appropriate vocational and academic programs for such individuals, provide them guidance on various psychotherapy methods, and create adjusted treatments for patients.

\begin{tabular}{|c|c|c|}
\hline \multicolumn{2}{|c|}{ Contribution Categories } & \multirow{2}{*}{$\begin{array}{l}\text { Author Initials } \\
\text { H.Y.C., M.B., C.E. }\end{array}$} \\
\hline \multirow{3}{*}{ Category 1} & Concept/Design & \\
\hline & Data acquisition & H.Y.C. \\
\hline & Data analysis/Interpretation & H.Y.C., M.B., C.E. \\
\hline \multirow{2}{*}{ Category 2} & Drafting manuscript & H.Y.C., M.B., C.E. \\
\hline & Critical revision of manuscript & C.E. \\
\hline Category 3 & Final approval and accountability & H.Y.C., M.B., C.E. \\
\hline \multirow{2}{*}{ Other } & Technical or material support & H.Y.C., M.B., C.E. \\
\hline & Supervision & M.B., C.E. \\
\hline
\end{tabular}

Ethics Committee Approval: The study protocol was approved by the Ethics Committee of Bakirkoy Training and Research Hospital for Psychiatry, Neurology and Neurosurgery (Turkey) (Date: 03.06.2014, Number: 403).

Informed Consent: Patient's were informed and written informed consent was obtained.

Peer-review: Externally peer-reviewed.

Conflict of Interest: No conflict of interest was declared by the authors.

Financial Disclosure: No financial disclosure was declared by the authors.

\section{REFERENCES}

1. Hall WD, Lynskey M. Is cannabis a gateway drug? Testing hypotheses about the relationship between cannabis use and the use of other illicit drugs. Drug Alcohol Rev 2005; 24:39-48.

2. Copeland J, Pokorski I. Progress toward pharmacotherapies for cannabis-use disorder: an evidence-based review. Subst Abuse Rehabil 2016; 7:41-53.

3. Evren C, Yilmaz Cengel H, Bozkurt M, Evren B, Umut G, Agachanli R. Psychometric properties of the Turkish versions of the Cannabis Use Problems Identification Test (CUPIT) and the adult Cannabis Problems Questionnaire (CPQ). J Psychoactive Drugs 2017; 49:83-89.

4. Evren C, Bozkurt M. Synthetic cannabinoids: crisis of the decade. Dusunen Adam The Journal of Psychiatry and Neurological Sciences 2013; 26:1-11.

5. Dresen S, Ferreirós N, Pütz M, Westphal F, Zimmermann R, Auwärter V. Monitoring of herbal mixtures potentially containing synthetic cannabinoids as psychoactive compounds. J Mass Spectrom 2010; 45:1186-1194.

6. European Monitoring Centre for Drugs and Drug Addiction (EMCDDA). New psychoactive substances in Europe: An update from the EU Early Warning System. https://www.emcdda.europa. eu/system/files/publications/65/TD0415135ENN.pdf. Accesed October 31, 2020.
7. Gurdal F, Asirdizer M, Aker RG, Korkut S, Gocer Y, Kucukibrahimoglu EE, et al. Review of detection frequency and type of synthetic cannabinoids in herbal compounds analyzed by Istanbul Narcotic Department of the Council of Forensic Medicine, Turkey. J Forensic Leg Med 2013; 20:667-672.

8. Schneir AB, Cullen J, Ly BT. "Spice" girls: synthetic cannabinoid intoxication. J Emerg Med 2011; 40:296-299.

9. Vardakou I, Pistos C, Spiliopoulou Ch. Spice drugs as a new trend: mode of action, identification and legislation. Toxicol Lett 2010; 197:157-162.

10. Castaneto MS, Gorelick DA, Desrosiers NA, Hartman RL, Pirard S, Huestis MA. Synthetic cannabinoids: epidemiology, pharmacodynamics, and clinical implications. Drug Alcohol Depend 2014; 144:12-41.

11. Demirakca T, Sartorius A, Ende G, Meyer N, Welzel H, Skopp $\mathrm{G}$, et al. Diminished gray matter in the hippocampus of cannabis users: possible protective effects of cannabidiol. Drug Alcohol Depend 2011; 114:242-245.

12. Hoyte CO, Jacob J, Monte AA, Al-Jumaan M, Bronstein AC, Heard KJ. A characterization of synthetic cannabinoid exposures reported to the National Poison Data System in 2010. Ann Emerg Med 2012; 60:435-438.

13. Perry JL, Carroll ME. The role of impulsive behavior in drug abuse. Psychopharmacology (Berl) 2008; 200:1-26.

14. de Wit H. Impulsivity as a determinant and consequence of drug use: a review of underlying processes. Addict Biol 2009; 14:22-31.

15. Patton JH, Stanford MS, Barratt ES. Factor structure of the Barratt impulsiveness scale. J Clin Psychol 1995; 51:768-774.

16. Cengel HY, Bozkurt M, Evren C, Umut G, Keskinkilic C, Agachanli R. Evaluation of cognitive functions in individuals with synthetic cannabinoid use disorder and comparison to individuals with cannabis use disorder. Psychiatry Res 2018; 262:46-54.

17. Gulec H, Tamam L, Gulec MY, Turhan M, Karakus G, Zengin M, Stanford MS. Psychometric Properties of the Turkish Version of the Barratt. Bull Clin Psychopharmacol 2008; 18:251-258.

18. Bashford J, Flett R, Copeland J. The Cannabis Use Problems Identification Test (CUPIT): development, reliability, concurrent and predictive validity among adolescents and adults. Addiction 2010; 105:615-625.

19. Drummond DC. The relationship between alcohol dependence and alcohol-related problems in a clinical population. Br J Addict 1990; 85:357-366.

20. Allsop DJ, Norberg MM, Copeland J, Fu S, Budney AJ. The Cannabis Withdrawal Scale development: patterns and predictors of cannabis withdrawal and distress. Drug Alcohol Depend 2011; 119:123-129.

21. Bozkurt M, Umut G, Evren C, Karabulut V. Clinical characteristics and laboratory test results of patients admitted to outpatient clinic for synthetic cannabinoid usage brief report. Dusunen Adam The Journal of Psychiatry and Neurological Sciences 2014; 27:328. 
22. Reinhard MA, Dewald-Kaufmann J, Wüstenberg T, Musil R, Barton BB, Jobst A, et al. The vicious circle of social exclusion and psychopathology: a systematic review of experimental ostracism research in psychiatric disorders. Eur Arch Psychiatry Clin Neurosci 2020; 270:521-532.

23. Fattore L. Synthetic Cannabinoids-further evidence supporting the relationship between cannabinoids and psychosis. Biol Psychiatry 2016; 79:539-548.

24. Patton AL, Chimalakonda KC, Moran CL, McCain KR, Radominska-Pandya A, James LP, et al. K2 toxicity: fatal case of psychiatric complications following AM2201 exposure. J Forensic Sci 2013; 58:1676-1680.

25. Waugh J, Najafi J, Hawkins L, Hill SL, Eddleston M, Vale JA, et al. Epidemiology and clinical features of toxicity following recreational use of synthetic cannabinoid receptor agonists: a report from the United Kingdom National Poisons Information Service. Clin Toxicol (Phila) 2016; 54:512-518.

26. Oliveira PMDS, Morais ASF, Madeira NGGF. Synthetic Cannabis Analogues and Suicidal Behavior: Case Report. J Addict Med 2017; 11:408-410.

27. Bassir Nia A, Medrano B, Perkel C, Galynker I, Hurd YL. Psychiatric comorbidity associated with synthetic cannabinoid use compared to cannabis. J Psychopharmacol 2016; 30:13211330

28. Clayton HB, Lowry R, Ashley C, Wolkin A, Grant AM. Health risk behaviors with synthetic cannabinoids versus marijuana. Pediatrics 2017; 139:e20162675.

29. Oznur T, Oznur H, Bolu A, Atik S, Akgun A, Kaymak S, et al. Relationship between suicide attempts and synthetic cannabinoids in adjustment disorder. Dusunen Adam J Psychiatry Neurol Sci 2018; 31:258-264.

30. Kamijo Y, Takai M, Fujita Y, Hirose Y, Iwasaki Y, Ishihara S, et al. A multicenter retrospective survey of poisoning after consumption of products containing synthetic chemicals in Japan. Intern Med 2014; 53:2439-2445.

31. Ashton $\mathrm{CH}$, Moore PB. Endocannabinoid system dysfunction in mood and related disorders. Acta Psychiatr Scand 2011; 124:250261.

32. Carli V, Jovanović N, Podlesek A, Roy A, Rihmer Z, Maggi S, et al. The role of impulsivity in self-mutilators, suicide ideators and suicide attempters - a study of 1265 male incarcerated individuals. J Affect Disord 2010; 123:116-122.

33. Ergelen M, Yalçın M, Bilici R. The comparison of violence, and the relationship with childhood trauma in Turkish men with alcohol, opiate, and synthetic cannabinoid use disorder. Neuropsychiatr Dis Treat 2018; 14:3169-3178.

34. Perry JL, Carroll ME. The role of impulsive behavior in drug abuse. Psychopharmacology (Berl) 2008; 200:1-26.

35. Cohen K, Kapitány-Fövény M, Mama Y, Arieli M, Rosca P, Demetrovics Z, et al. The effects of synthetic cannabinoids on executive function. Psychopharmacology (Berl) 2017; 234:11211134.

36. Verdejo-García A, Lawrence AJ, Clark L. Impulsivity as a vulnerability marker for substance-use disorders: review of findings from high-risk research, problem gamblers and genetic association studies. Neurosci Biobehav Rev 2008; 32:777-810.

37. Ramírez JM, Andreu JM. Aggression, and some related psychological constructs (anger, hostility, and impulsivity); some comments from a research project. Neurosci Biobehav Rev 2006; 30:276-291.

38. Dick DM, Smith G, Olausson P, Mitchell SH, Leeman RF, O’Malley SS, et al. Understanding the construct of impulsivity and its relationship to alcohol use disorders. Addict Biol 2010; 15:217-226.

39. Bozkurt M, Evren C, Yilmaz A, Can Y, Cetingok S. Aggression and impulsivity in different groups of alcohol and heroin dependent inpatient men. Bulletin Clin Psychopharmacol 2013; 23:335-344. 